\title{
On Constrained Open-World Probabilistic Databases
}

\author{
Tal Friedman and Guy Van den Broeck \\ University of California, Los Angeles \\ $\{$ tal, guyvdb\}@cs.ucla.edu
}

\begin{abstract}
Increasing amounts of available data have led to a heightened need for representing large-scale probabilistic knowledge bases. One approach is to use a probabilistic database, a model with strong assumptions that allow for efficiently answering many interesting queries. Recent work on openworld probabilistic databases strengthens the semantics of these probabilistic databases by discarding the assumption that any information not present in the data must be false. While intuitive, these semantics are not sufficiently precise to give reasonable answers to queries. We propose overcoming these issues by using constraints to restrict this open world. We provide an algorithm for one class of queries, and establish a basic hardness result for another. Finally, we propose an efficient and tight approximation for a large class of queries.
\end{abstract}

\section{Introduction}

An ubiquitous pursuit in the study of knowledge base representation is the search for a model that can represent uncertainty while simultaneously answering interesting queries efficiently. The key underlying challenge is that these goals are at odds with each other. Modelling uncertainty requires additional model complexity. At the same time, the ability to answer meaningful queries usually demands restrictive model assumptions. Both of these properties are at odds with the key limiting factor of tractability: success in the first two goals is not nearly as impactful if it is not achieved efficiently. Unfortunately, probabilistic reasoning is often computationally hard, even on databases [Roth, 1996; Dalvi and Suciu, 2012].

One approach towards achieving this goal is to begin with a simple model such a probabilistic database (PDB) [Suciu et al., 2011; Van den Broeck and Suciu, 2017]. A PDB models uncertainty, but is inherently simple and makes very strong independence assumptions and closed-world assumptions allowing for tractability on a very large class of queries [Dalvi and Suciu, 2007; Dalvi and Suciu, 2012]. However, PDBs can fall short under non-ideal circumstances, as their semantics are brittle to incomplete knowledge bases [Ceylan et al., 2016].
To bring PDBs closer to the desired goal, Ceylan et al. 2016 propose open-world probabilistic databases (OpenPDB), wherein the semantics of a PDB are strengthened to relax the closed-world assumption. While OpenPDBs maintain a large class of tractable queries, their semantics are so relaxed these queries lose their precision: they model further uncertainty, but in exchange give less useful query answers.

In this work, we aim to overcome these querying challenges, while simultaneously maintaining the degree of uncertainty modeled by OpenPDBs. To achieve this, we propose further strengthening the semantics of OpenPDBs by constraining the mean probability allowed for a relation. These constraints work at the schematic level, meaning no additional per-item information is required. They are practically motivated by knowledge of summary statistics, of how many tuples we expect to be true. A theoretical analysis shows that, despite their simplicity, such constraints fundamentally change the difficulty landscape of queries, leading us to propose a general-purpose approximation scheme.

The rest of the paper is organized as follows: Section 2 provides necessary background on relational logic and PDBs, as well as an introduction to OpenPDBs. Section 3 motivates and introduces our construction for constraining OpenPDBs. Section 4 analyses exact solutions subject to these constraints, providing a class of tractable queries along with an algorithm. It also shows that the problem is in general hard, even in some cases where standard PDB queries are tractable. Section 5 investigates an efficient and provably bounded approximation scheme. Section 6 discusses our findings, and summarizes interesting directions that we leave as open problems.

\section{Background}

This section provides background and motivation for probabilistic databases and their open-world counterparts. Notation and definitions are adapted from Ceylan et al. 2016.

\subsection{Relational Logic and Databases}

We now describe necessary background from function-free finite-domain first-order logic. An atom $R\left(x_{1}, x_{2}, \ldots, x_{n}\right)$ consists of a predicate $R$ of arity $n$, together with $n$ arguments. These arguments can either be constants or variables. A ground atom is an atom that contains no variables. A formula is a series of atoms combined with conjunctions $(\wedge)$ 
Proceedings of the Twenty-Eighth International Joint Conference on Artificial Intelligence (IJCAI-19)

\begin{tabular}{lll}
\hline \multicolumn{1}{c}{ Scientist } & & \multicolumn{2}{c}{ CoAuthor } \\
$\begin{array}{l}\text { Einstein } \\
\text { Erdős }\end{array}$ & & Einstein Erdős \\
von Neumann & & Erdős $\quad$ von Neumann \\
\cline { 2 - 3 } & &
\end{tabular}

Figure 1: Example relational database. Notice that the first row of the right table corresponds to the atom CoAuthor(Einstein, Erdős).

\begin{tabular}{l|c}
\hline \multicolumn{1}{c|}{ Scientist } & $p$ \\
\hline Einstein & 0.8 \\
Erdős & 0.8 \\
von Neumann & 0.9 \\
Shakespeare & 0.2 \\
\hline
\end{tabular}

\begin{tabular}{ll|c}
\hline \multicolumn{2}{c|}{ CoAuthor } & $p$ \\
\hline Einstein & Erdős & 0.8 \\
Erdős & von Neumann & 0.9 \\
von Neumann & Einstein & 0.5 \\
\hline
\end{tabular}

Figure 2: Example probabilistic database. Tuples are now of the form $\langle t: p\rangle$ where $p$ is the probability of the tuple $t$ being present.

or disjunctions $(\vee)$, and with quantifiers $\forall, \exists$. A substitution $\mathrm{Q}[x / t]$ replaces all occurences of $x$ by $t$ in a formula $\mathrm{Q}$.

A relational vocabulary $\sigma$ is comprised of a set of predicates $\mathcal{R}$ and a domain $\mathcal{D}$. Using the Herbrand semantics [Hinrichs and Genesereth, 2006], the Herbrand base of $\sigma$ is the set of all ground atoms possible given $\mathcal{R}$ and $\mathcal{D}$. A $\sigma$ interpretation $\omega$ is then an assignment of truth values to every element of the Herbrand base of $\sigma$. We say that $\omega$ models a formula $Q$ whenever $\omega$ satisfies $Q$. This is denoted by $\omega=Q$.

A reasonable starting point for the target knowledge base to construct would be to use a traditional relational database. Using the standard model-theoretic view [Abiteboul et al., 1995], a relational database for a vocabulary $\sigma$ is a $\sigma$ interpretation $\omega$. Less formally, a relational database consists of a series of relations, each of which corresponds to a predicate. Each relation consists of a series of rows, also called tuples, each of which corresponds to an atom of the predicate being true. Any atom not appearing as a row in the relation is considered to be false, following the closed-world assumption [Reiter, 1981]. Figure 1 shows an example database.

\subsection{Probabilistic Databases}

Despite the success of relational databases, their deterministic nature leads to a few shortcomings. A common way to gather a large knowledge base is to apply some sort of statistical model [Carlson et al., 2010; Suchanek et al., 2007; Peters et al., 2014; Dong et al., 2014] which returns a probability value for potential tuples. Adapting the output of such a model to a relational database involves thresholding on the probability value, discarding valuable information along the way. A probabilistic database (PDB) circumvents this problem by assigning each tuple a probability.

Definition 1. A (tuple-independent) probabilistic database $\mathcal{P}$ for a vocabulary $\sigma$ is a finite set of tuples of the form $\langle t: p\rangle$ where $t$ is a $\sigma$-atom and $p \in[0,1]$. Furthermore, each $t$ can appear at most once.

Given such a collection of tuples and their probabilities, we are now going to define a distribution over relational databases. The semantics of this distribution are given by treating each tuple as an independent random variable.
Definition 2. A probabilistic database $\mathcal{P}$ for vocabulary $\sigma$ induces a probability distribution over $\sigma$-interpretations $\omega$ :

$$
\begin{aligned}
& P_{\mathcal{P}}(\omega)=\prod_{t \in \omega} P_{\mathcal{P}}(t) \prod_{t \notin \omega}\left(1-P_{\mathcal{P}}(t)\right) \\
& \text { where } P_{\mathcal{P}}(t)= \begin{cases}p & \text { if }\langle t: p\rangle \in \mathcal{P} \\
0 & \text { otherwise }\end{cases}
\end{aligned}
$$

Notice this last statement is again making the closed-world assumption: any tuple that we have no information about is assigned probability zero. Figure 2 shows an example PDB.

\section{Probabilistic Queries}

In relational databases, the fundamental task we are interested in solving is how to answer queries. The same is true for probabilistic databases, with the only difference being that we are now interested in probabilities over queries. In particular, we are interested in queries that are fully quantified also known as Boolean queries. On a relational database, this corresponds to a query that has an answer of True or False.

For example, on the database given in Figure 1, we might ask if there is a scientist who is a coauthor:

$$
\mathrm{Q}_{1}=\exists x \cdot \exists y \cdot S(x) \wedge C o A(x, y)
$$

If we instead asked this query of the probabilistic database in Figure 2, we would be computing the probability by summing over the worlds in which the query is true:

$$
P\left(\mathrm{Q}_{1}\right)=\sum_{\omega \models \mathrm{Q}_{1}} P_{\mathcal{P}}(\omega)
$$

Queries of this form that are a conjunction of atoms are called conjunctive queries. They are commonly shortened as:

$$
\mathrm{Q}_{1}=S(x), \operatorname{CoA}(x, y) \text {. }
$$

A disjunction of conjunctive queries is known as a union of conjunctive queries (UCQ). UCQs have been shown to live in a dichotomy of efficient evaluation [Dalvi and Suciu, 2012]: computing the probability of a UCQ is either polynomial in the size of the database, or it is \#P-hard. This property can be checked through the syntax of a query, and we say that a UCQ is safe if it admits efficient evaluation. In the literature of probabilistic databases [Suciu et al., 2011; Dalvi and Suciu, 2012], as well as throughout the rest of this paper, UCQs are the primary query object studied.

\section{Efficient Query Evaluation}

For probabilistic databases to be useful, we need to be able to efficiently compute the probabilities of queries: we now describe how to do this. Algorithm 1 does this in polynomial time for all queries that can be computed efficiently (known as safe queries). We now explain the steps in further detail.

We begin with the assumption that $\mathrm{Q}$ has been processed to not contain any constant symbols, and that all variables appear in the same order in repeated predicate occurences in Q. These preprocessing steps are known as shattering and ranking respectively, and can be done efficiently [Dalvi and Suciu, 2012].

Step 0 covers the base case where $Q$ is simple a tuple, so it looks it up in $\mathcal{P}$. Step 1 attemps to rewrite the UCQ into 


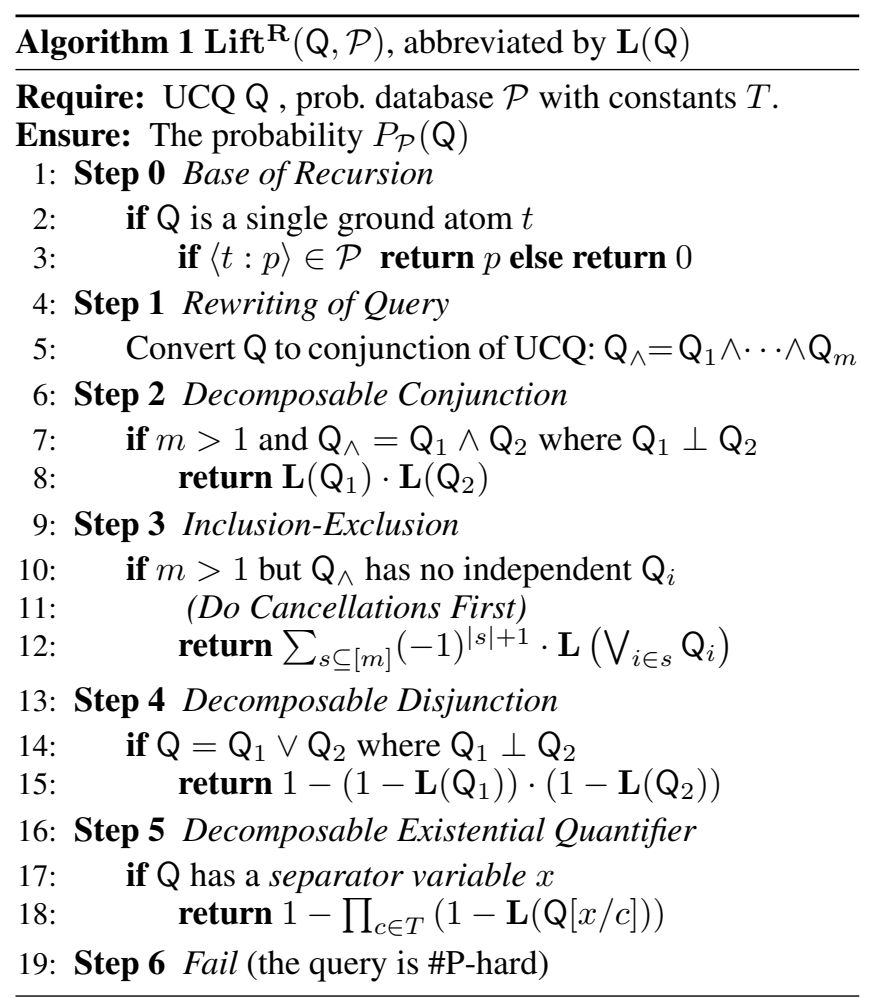

a conjunction of UCQs to find decomposable parts. For example, the UCQ $(R(x) \wedge S(y, z)) \vee(S(x, y) \wedge T(x))$ can be written as the conjunction of $(R(x)) \vee(S(x, y) \wedge T(x))$ and $(S(y, z)) \vee(S(x, y) \wedge T(x))$. When multiple conjuncts are found this way, there are two options. If they are symbolically independent (share no symbols, denoted $\perp$ ), then Step 2 applies independence and recurse. Otherwise, Step 3 recurses using the inclusion-exclusion principle, performing cancellations first to maintain efficiency [Dalvi and Suciu, 2012]. If there is only a single UCQ after rewriting, Step 4 tries to split it into independent parts, applying independence and recursing if anything is found.

Next, Step 5 searches for a separator variable, one which appears in every atom in $\mathrm{Q}$. If $x$ is a separator variable for $\mathrm{Q}$, and $a, b$ are different constants in the domain of $x$, this means that $\mathrm{Q}[x / a]$ and $\mathrm{Q}[x / b]$ are independent. This independence is again recursively exploited. Finally, if Step 6 is reached, then the algorithm has failed and the query cannot be computed efficiently [Dalvi and Suciu, 2012].

\subsection{Open-World Probabilistic Databases}

In the context of automatically constructing a knowledge base, as is done in for example NELL [Carlson et al., 2010] or Google's Knowledge Vault [Dong et al., 2014], making the closed-world assumption is conceptually unreasonable. Conversely, it is also not feasible to include all possible tuples and their probabilities in the knowledge base. The resulting difficulty is that there are an enormous number of probabilistic facts that can be scraped from the internet, and by definition these tools will keep only those with the very highest probability. As a result, knowledge bases like NELL [Carlson et al., 2010], PaleoDeepDive [Peters et al., 2014], and YAGO
[Suchanek et al., 2007] consist almost entirely of probabilities above 0.95 .

This tells us that the knowledge base we are looking at is fundamentally incomplete. In response to this problem, Ceylan et al. 2016 propose the notion of a completion for a probabilistic database.

Definition 3. A $\lambda$-completion of a probabilistic database $\mathcal{P}$ is another probabilistic database obtained as follows. For each atom $t$ that does not appear in $\mathcal{P}$, we add tuple $\langle t: p\rangle$ to $\mathcal{P}$ for some $p \in[0, \lambda]$.

Then, we can define the open world of possible databases in terms of the set of distributions induced by all completions.

Definition 4. An open-world probabilistic database (OpenPDB) is a pair $\mathcal{G}=(\mathcal{P}, \lambda)$, where $\mathcal{P}$ is a probabilistic database and $\lambda \in[0,1]$. $\mathcal{G}$ induces a set of probability distributions $K_{\mathcal{G}}$ such that a distribution $\mathrm{P}$ belongs to $K_{\mathcal{G}}$ iff $\mathrm{P}$ is induced by some $\lambda$-completion of probabilistic database $\mathcal{P}$.

\section{Open-World Queries}

OpenPDBs specify a set of probability distributions rather than a single one, meaning that a given query produces a set of possible probabilities rather than a single one. We focus on computing the minimum and maximum possible probability values that can be achieved by completing the database.

Definition 5. The probability interval of a Boolean query $\mathrm{Q}$ in OpenPDB $\mathcal{G}$ is $K_{\mathcal{G}}(\mathrm{Q})=\left[\underline{P}_{\mathcal{G}}(\mathrm{Q}), \bar{P}_{\mathcal{G}}(\mathrm{Q})\right]$, where

$$
\underline{P}_{\mathcal{G}}(\mathrm{Q})=\min _{P \in K_{\mathcal{G}}} P(\mathrm{Q}) \quad \bar{P}_{\mathcal{G}}(\mathrm{Q})=\max _{P \in K_{\mathcal{G}}} P(\mathrm{Q})
$$

In general, computing the probability interval for some first-order $Q$ is not tractable. As observed in Ceylan et al. 2016, however, the situation is different for UCQ queries, because they are monotone (they contain no negations). For UCQs, the upper and lower bounds are given respectively by the full completion (where all unknown probabilities are $\lambda$ ), and the closed world database. This is a direct result of the fact that OpenPDBs form a credal set: a closed convex set of probability measures, meaning that probability bounds always come from extreme points [Cozman, 2000].

Furthermore, Ceylan et al. 2016 also provide an algorithm for efficiently computing this upper bound corresponding to a full completion, and show that it works whenever the UCQ is safe.

\section{Mean-Constrained Completions}

This section motivates the need to strengthen the OpenPDB semantics, and introduces our novel probabilistic data model.

\subsection{Motivation}

The ability to perform efficient query evaluation provides an appealing case for OpenPDBs. They give a more reasonable semantics, better matching their use, and for a large class of queries they come at no extra cost in comparison to traditional PDBs. However, in practice computing an upper bound in this way tends to give results very close to 1 . Intuitively, this makes sense: our upper bound comes from simultaneously 


\begin{tabular}{llll}
\hline Query & CW & OW & CoOW \\
\hline $\operatorname{LiLA}(x), S(x)$ & 0 & $1-10^{-290}$ & $1-10^{-15}$ \\
$\operatorname{LiSpr}(x), S(x)$ & 0 & $1-10^{-191}$ & 0.96 \\
\hline
\end{tabular}

Table 1: Comparison of upper bounds for the same query and database with different model assumptions: Closed-World (CW), Open-World (OW), and Constrained Open-World (CoOW).

assuming that every possible missing atom has some reasonable probability. While such a bound is easy to compute, it is too strong of a relaxation of the closed-world assumption.

Recall the motivation for the initial OpenPDB semantics: statistical knowledge base construction (KBC) tools store only the most likely extracted tuples [Ceylan et al., 2016]. The $\lambda$ parameter in OpenPDBs is designed to account for this, representing an upper bound on the probability of unobserved tuples. However, this discards other information potentially collected by the KBC system: for example, suppose that a table in our database describes whether or not a person is a scientist. The OpenPDB model will account for the fact that many of the people we discard have a non-zero chance of being a scientist, but it will not take into account the fact that our KBC system observes that fewer than $1 \%$ of the population are scientists.

In order to consider a restricted subset of completions representing reasonable situations, we propose directly incorporating these summary statistics. Specifically, we place constraints on the overall probability of a relation across the entire population. In the scientist example, our model only considers completions in which the probability mass of people being scientists totals less than $1 \%$. This allows us to include more information at the domain level, without having more information about each individual.

Example. To illustrate the effect this has, consider a schema in which we have 3 relations: $\operatorname{LiLA}(x)$ denoting whether one lives in Los Angeles, $\operatorname{LiSpr}(x)$ denoting whether one lives in Springfield, and $S(x)$ denoting whether one is a scientist. Using a vocabulary of 500 people where each person is present in at most one relation, Table 1 shows the resulting upper probability bound under different model assumptions, where the constrained open-world restricts at most $50 \%$ of mass on LiLA, $5 \%$ on $S$, and $0.5 \%$ on LiSpr. In particular, notice how extreme the difference is in upper bound with and without constraints being imposed. The closed-world probability of both of these queries is always 0 , as each person in our database only has a known probability for at most one relation. It is clear that of these three options, the constrained open-world is the most reasonable - the rest of this section formalizes this idea and investigates the resulting properties.

\subsection{Formalization}

We begin here by defining mean-based constraints, before examining some immediate observations about the structure of the resulting constrained database.

Definition 6. Suppose we have a PDB $\mathcal{P}$, and let $\operatorname{Tup}(R) \subseteq$ $\mathcal{P}$ be the set of probabilistic tuples in relation $R$. Let $\bar{p}$ be a probability threshold. Then a mean tuple probability constraint (MTP constraint) $\varphi$ is a linear constraint of the form

$$
\bar{p} \geq \frac{1}{|\operatorname{Tup}(R)|} \sum_{\langle t: p\rangle \in \operatorname{Tup}(R)} p
$$

Definition 7. We say that a $\lambda$-completion is $\varphi$-constrained if the $\lambda$-completed database satisfies $\operatorname{MTP} \varphi$. If it satisfies all of $\Phi=\left(\varphi_{1}, \varphi_{2}, \ldots, \varphi_{n}\right)$, then we say it is $\Phi$-constrained.

Being $\varphi$-constrained is not a property of OpenPDBs, but of their PDB completions. Hence, we are interested in the subset of completions that satisfy this property.

Definition 8. An OpenPDB $\mathcal{G}=(\mathcal{P}, \lambda)$ together with MTP constraints $\Phi$ induces a set of probability distributions $K_{\mathcal{G}}^{\Phi}$, where distribution $\mathrm{P}$ belongs to $K_{\mathcal{G}}^{\Phi}$ iff $\mathrm{P}$ is induced by some $\Phi$-constrained $\lambda$-completion of $\mathcal{P}$.

Much like with standard OpenPDBs, for a Boolean query $\mathrm{Q}$ we are interested in computing bounds on $P(\mathrm{Q})$.

Definition 9. The probability interval of a Boolean query $\mathrm{Q}$ in OpenPDB $\mathcal{G}$ with MTP constraints $\Phi$ is $K_{\mathcal{G}}^{\Phi}(\mathrm{Q})=$ $\left[\underline{P}_{\mathcal{G}}^{\Phi}(\mathrm{Q}), \bar{P}_{\mathcal{G}}^{\Phi}(\mathrm{Q})\right]$, where

$$
\underline{P}_{\mathcal{G}}^{\Phi}(\mathrm{Q})=\min _{P \in K_{\mathcal{G}}^{\Phi}} P(\mathrm{Q}) ; \quad \bar{P}_{\mathcal{G}}^{\Phi}(\mathrm{Q})=\max _{P \in K_{\mathcal{G}}^{\Phi}} P(\mathrm{Q}) .
$$

\subsection{Completion Properties}

A necessary property of OpenPDBs for efficient query evaluation is that they are credal - this is what allows us to consider only a finite subset of possible completions. MTPconstrained OpenPDBs maintain this property. ${ }^{1}$

Proposition 1. Suppose we have an OpenPDB $\mathcal{G}$ together with MTP constraints $\Phi$. Then the induced set of probability distributions $K_{\mathcal{G}}^{\Phi}$ is credal.

This property allows us to examine only a finite subset of configurations when looking at potential completions, since query probability bounds of a credal set are always achieved at points of extrema [Cozman, 2000]. Next, we would like to characterize these points of extrema, by showing that the number of tuples not on their own individual boundaries (that is, 0 or $\lambda$ ) is given by the number of MTP constraints.

Theorem 2. Suppose we have an OpenPDB $\mathcal{G}=(\mathcal{P}, \lambda)$ with $M T P$ constraints $\Phi$, and a UCQ $Q$. Then there exists a $\Phi$ constrained $\lambda$-completion $\mathcal{P}^{\prime}$ for which $P_{\mathcal{P}^{\prime}}(\mathrm{Q})=\bar{P}_{\mathcal{G}}^{\Phi}(\mathrm{Q})$ and that contains some $\mathcal{T} \subseteq \mathcal{P}^{\prime} \backslash \mathcal{P}$ such that $|\mathcal{T}| \leq|\Phi|$, and

$$
\begin{aligned}
\forall\langle t: p\rangle \in \mathcal{T}: & p \in[0, \lambda], \text { and } \\
\forall\langle t: p\rangle \in\left(\mathcal{P}^{\prime} \backslash \mathcal{P}\right) \backslash \mathcal{T}: & p \in\{0, \lambda\} .
\end{aligned}
$$

That is, our upper bound is given by a completion that has at most $|\Phi|$ added tuples with probability not exactly 0 or $\lambda$. Intuitively, each MTP constraint contributes a single non-boundary tuple, which can be thought of as the "leftover" probability mass once the rest has been assigned in full.

\footnotetext{
${ }^{1}$ Proofs of all theorems and lemmas are available in appendix of the full version of the paper at http://starai.cs.ucla.edu/papers/ FriedmanIJCAI19.pdf
} 
This insight allows us to treat MTP query evaluation as a combinatorial optimization problem for the rest of this paper. Thus, we only consider the case where achieving the mean tuple probability exactly leaves us with every individual tuple at its boundary. To see that we can do this, we observe that Theorem 2 leaves a single tuple per MTP constraint not necessarily on the boundary. But this tuple can always be forced to be on the boundary by very slightly increasing the mean $\bar{p}$ of the constraint, as follows.

Corollary 3. Suppose we have an OpenPDB $\mathcal{G}=(\mathcal{P}, \lambda)$ with MTP constraints $\Phi$, and a UCQ Q. Suppose further that each relation in $\mathcal{G}$ has at most 1 constraint in $\Phi$, and that each constraint allows adding open-world probability mass exactly divisible by $\lambda$. Then there exists a $\Phi$-constrained $\lambda$ completion $\mathcal{P}^{\prime}$, where $K_{\mathcal{G}}^{\Phi}(\mathrm{Q})=\left[P_{\mathcal{P}}(\mathrm{Q}), P_{\mathcal{P}^{\prime}}(\mathrm{Q})\right]$, and

$$
\forall\langle t: p\rangle \in \mathcal{P}^{\prime} \backslash \mathcal{P}: p \in\{0, \lambda\} .
$$

Our investigation into the algorithmic properties of MTP query evaluation will be focused on constraining a single relation, subject to a single combinatorial budget constraint.

\section{Exact MTP Query Evaluation}

With Section 3 formalizing MTP constraints and showing that computing upper bounds subject to MTP constraints is a combinatorial problem of choosing which $\lambda$-probability tuples to add in the completion, we now investigate exact solutions. With this now being a combinatorial problem, we slightly change our terminology: "adding" an open-world tuple $t$ to a relation means we consider only completions where $P(t)=\lambda$, and a "budget" $b$ for a relation means we can add up to $b$ tuples while still satisfying the MTP constraint.

\subsection{An Algorithm for Inversion-Free Queries}

We begin by describing a class of queries that admits polytime evaluation subject to an MTP constraint. We first need to define some syntactic properties of queries.

Definition 10. Let $\mathrm{Q}$ be a conjunctive query, and let $a t(x)$ denote the set of relations containing variable $x$. We say that $\mathrm{Q}$ is hierarchical if for any $x, y$, we have either $a t(x) \subseteq a t(y)$, $a t(y) \subseteq a t(x)$, or $a t(x) \cap a t(y)=\emptyset$.

Intuitively, a conjunctive query being hierarchical indicates that it can either be separated into independent parts (the $a t(x) \cap a t(y)=\emptyset$ case), or there is some variable that appears in every atom. This simple syntactic property is the basis for determining whether query evaluation on a conjunctive query can be done in polynomial time [Dalvi and Suciu, 2007]. We can further expand on this definition in the context of UCQs.

Definition 11. A UCQ $Q$ is inversion-free if each of its disjuncts is hierarchical, and they all share the same hierarchy. ${ }^{2}$ If $Q$ is not inversion-free, we say that it has an inversion.

Inversion-free queries represent an especially tractable class of queries for general inference. Since they are hierarchical, they are also safe, meaning query evaluation is efficient. Moreover, they precisely characterize the class of

\footnotetext{
${ }^{2}$ See Jha and Suciu 2011 for a more detailed definition.
}

queries that support compilation to a tractable form for performing more complex queries, such as computing any joint distribution [Jha and Suciu, 2011]. This query class remains tractable under MTP constraints.

Theorem 4. For any inversion-free query $\mathrm{Q}$, evaluating the probability $\bar{P}_{\mathcal{G}}^{\Phi}(\mathrm{Q})$ subject to an MTP constraint is in PTIME.

In order to prove Theorem 4, we provide a polytime algorithm for MTP query evaluation on inversion-free queries. As with OpenPDBs, our algorithm depends on Algorithm 1, the standard lifted inference algorithm for PDBs that was discussed in Section 2.

We now present an algorithm for doing exact MTP query evaluation on inversion-free queries. For brevity, we present the case of a binary relation; the general case follows similarly and can be found in appendix. Suppose that we have a probabilistic database $\mathcal{P}$, a domain $T$ of constants denoted $c$, a query $\mathrm{Q}$, and an MTP constraint on relation $R(x, y)$ allowing us to add exactly $b$ tuples with probability $\lambda$. Suppose that Q immediately reaches Step 5 of Algorithm 1 (other steps will be discussed later), implying that $x$ and $y$ are unique variables in the query. We let $A\left(c_{x}, c_{y}, b\right)$ denote the upper query probability of $\mathrm{Q}\left(x / c_{x}, y / c_{y}\right)$ subject to an MTP constraint allowing budget $b$ on $R$ restricted to $x=c_{x}, y=c_{y}$. That is, $A$ tells us the highest probability we can achieve for a partial assignment given a fixed budget. Observe that we can compute all entries of $A$ using a slight modification of Algorithm 1 where we compute probabilities with and without each added tuple. This will take time polynomial in $|T|$.

Next, we impose an ordering $c_{1}, \ldots, c_{|T|}$ on the domain. Then we let $D\left(j, c_{y}, b\right)$ denote the upper query probability of

$$
\bigvee_{c \in\left\{c_{1}, \ldots c_{j}\right\}} \mathrm{Q}\left(x / c, y / c_{y}\right)
$$

with a budget of $b$ on the relevant portions of $R$. Then $D\left(|T|, c_{y}, b\right)$ considers all possible substitutions in our first index, meaning we have effectively removed a variable. Doing this repeatedly would allow us to perform exact MTP query evaluation. However, $D$ is non-trivial to compute, and cannot be done by simply modifying Algorithm 1. Instead, we observe the following recurrence:

$$
\begin{aligned}
D\left(j+1, y / c_{y}, b\right) & = \\
\max _{k \in\{1, \ldots, b\}} 1- & \left(1-D\left(j, y / c_{y}, b-k\right)\right) \\
\cdot & \left(1-A\left(x / c_{j+1}, y / c_{y}, k\right)\right)
\end{aligned}
$$

Intuitively, this recurrence says that since the tuples from each fixed constant are all independent, we do not need to store which budget configuration on the first $j$ constants got us our optimal solution. Thus, when we add the $j+1$ th constant, we just need to check each possible value we could assign to our new constant, and see which gives the overall highest probability. This recurrence can be implemented efficiently, yielding a dynamic programming algorithm that runs in time polynomial in the domain size and budget.

Finally, we would like to generalize this algorithm beyond the assumption that $Q$ immediately reaches Step 5 of Algorithm 1. Looking at other cases, we see that Steps 0 and 1 
have no effect on this recurrence, and that Steps 2 and 4 correspond to multiplicative factors. For a query that reaches Step 3 (inclusion-exclusion), we need to construct such $A$ and $D$ for each term in the inclusion-exclusion sum, and follow the analogous recurrence.

Notice that the modified algorithm would only work in the case where we can always pick a common variable for all sub-queries to do dynamic programming on - that is, when the query is inversion-free, as was our assumption. If the subcalls generated by inclusion-exclusion do not share a common variable hierarchy, and thus an order for using our dynamic programming algorithm, we suffer an exponential blowup.

\subsection{Queries with Inversion}

We now show that allowing for inversions in safe queries can cause MTP query evaluation to become NP-hard. Interestingly, this means that MTP constraints fundamentally change the difficulty landscape of query evaluation.

To show this, we investigate the following UCQ query.

$$
\begin{aligned}
M_{0}=\exists x \exists y \exists z & (R(x, y, z) \wedge U(x)) \vee(R(x, y, z) \wedge V(y)) \\
& \vee(R(x, y, z) \wedge W(z)) \vee(U(x) \wedge V(y)) \\
& \vee(U(x) \wedge W(z)) \vee(V(y) \wedge W(z))
\end{aligned}
$$

A key observation here is that the query $M_{0}$ is a safe UCQ. That is, if we ignore constraints and evaluate it subject to the closed- or open-world semantics, computing the probability of the query would be polynomial in the size of the database. We now show that this is not the case for open-world query evaluation subject to a single MTP constraint on $R$.

Theorem 5. Evaluating the upper query probability bound $\bar{P}_{\mathcal{G}}^{\Phi}\left(M_{0}\right)$ subject to an MTP constraint $\Phi$ on $R$ is NP-hard.

The full proof of Theorem 5 can be found in appendix, showing a reduction from the NP-complete 3-dimensional matching problem to computing $\bar{P}_{\mathcal{G}}^{\Phi}\left(M_{0}\right)$ with an MTP constraint on $R$. It uses the following intuitive correspondence.

Definition 12. Let $X, Y, Z$ be finite disjoint sets representing nodes, and let $T \subseteq X \times Y \times Z$ be the set of available hyperedges. Then $M \subseteq T$ is a matching if for any distinct triples $\left(x_{1}, y_{1}, z_{1}\right) \in \bar{M},\left(x_{2}, y_{2}, z_{2}\right) \in M$, we have that $x_{1} \neq x_{2}, y_{1} \neq y_{2}, z_{1} \neq z_{2}$. The 3-dimensional matching decision problem is to determine for a given $X, Y, Z, T$ and positive integer $k$ if there exists a matching $M$ with $|M| \geq k$.

The set of available tuples for $R$ will correspond to all edges in $T$. The MTP constraint on $R$ forces a decision on which subset of $T$ to add to the database.

However, if we simply queried to maximize $P(R(x, y, z))$, this completion need not correspond to a matching. Instead, we have the disjunct $R(x, y, z) \wedge U(x)$ which is maximized when each tuple chosen from $R$ has a different $x$ value. Similar disjuncts for $y$ and $z$ ensure that the query is maximized when using distinct $y$ and $z$ values. Putting all of these together ensures that the query probability is maximized when the subset of tuples chosen to complete $R$ form a matching.

Finally, the last part of the query $(U(x) \wedge V(y)) \vee(U(x) \wedge$ $W(z)) \vee(V(y) \wedge W(z))$ ensures that inference on $M_{0}$ is tractable, but it is unaffected by the choice of tuples in $R$.

\section{Approximate MTP Query Evaluation}

With Section 4.2 answering definitively that a generalpurpose algorithm for evaluating MTP query bounds is unlikely to exist, even when restricted to safe queries, an approximation is the logical next step. We now restrict our discussion to situations where we constrain a single relation, and dig deeper into the properties of MTP constraints to show their submodular structure. We then exploit this property to achieve efficient bounds with guarantees.

\subsection{On the Submodularity of Adding Tuples}

To formally define and prove the submodular structure of the problem, we analyze query evaluation as a set function on adding tuples. We begin with a few relevant definitions.

Definition 13. Suppose that we have an OpenPDB $\mathcal{G}$, with an MTP constraint $\varphi$ on a single relation $R$, and we let $\mathbf{O}$ be the set of possible tuples we can add to $R$. Then the set query probability function $S_{\mathcal{P}, \mathrm{Q}}: 2^{\mathrm{O}} \rightarrow[0,1]$ is defined as

$$
S_{\mathcal{P}, \mathrm{Q}}(X)=P_{\mathcal{P} \cup\{\langle t: \lambda\rangle \mid t \in X\}}(\mathrm{Q}) .
$$

Intuitively, this function describes the probability of the query as a function of which open tuples have been added. It provides a way to reason about the combinatorial properties of this optimization problem. Observe that $S_{\mathcal{P}, \mathrm{Q}}(\emptyset)$ is the closed-world probability of the query, while $S_{\mathcal{P}, \mathrm{Q}}(\mathbf{O})$ is the open-world probability.

We want to show that $S_{\mathcal{P}, \mathrm{Q}}$ is a submodular set function.

Definition 14. A submodular set function is a function $f$ : $2^{\Omega} \rightarrow \mathbb{R}$ such that for every $X \subseteq Y \subseteq \Omega$, and every $x \in$ $\Omega \backslash Y$, we have that

$$
f(X \cup\{x\})-f(X) \geq f(Y \cup\{x\})-f(Y) .
$$

Theorem 6. The set query probability function $S_{\mathcal{P}, \mathrm{Q}}$ is submodular for any tuple independent probabilistic database $\mathcal{P}$ and UCQ query $\mathrm{Q}$ without self-joins.

This gives us the desired submodularity property, which we can exploit to build efficient approximation algorithms.

\subsection{From Submodularity to Approximation}

Given the knowledge that the probability of a safe query without self-joins is submodular in the completion of a single relation, we are now tasked with using this to construct an efficient approximation. Since we further know the probability is also monotone as we have restricted our language to UCQs, Nemhauser et al. 1978 tell us that we can get a $1-\frac{1}{e}$ approximation using a simple greedy algorithm. The final requirement to achieve the approximation described in Nemhauser et al. 1978 is that our set function must have the property that $f(\emptyset)=0$. This can be achieved in a straightforward manner as follows.

Definition 15. In the context of the set query probability function of Definition 13, the normalized set query probability function $S_{\mathcal{P}, \mathrm{Q}}^{\prime}: 2^{\mathrm{O}} \rightarrow[0,1]$ is defined as

$$
S_{\mathcal{P}, \mathrm{Q}}^{\prime}(X)=P_{\mathcal{P} \cup\{\langle t: \lambda\rangle \mid t \in X\}}(\mathrm{Q})-P_{\mathcal{P}}(\mathrm{Q}) .
$$

Proposition 7. Any normalized set query probability function $S_{\mathcal{P}, \mathrm{Q}}^{\prime}$ is monotone, submodular, and satifies $S_{\mathcal{P}, \mathrm{Q}}^{\prime}(\emptyset)=0$. 
By simply normalizing the set query probability function, we can now directly apply the greedy approximation described in Nemhauser et al. 1978. We slightly modify Algorithm 1 to efficiently compute the next best tuple to add based on the current database, and add it. This is repeated until adding another tuple would violate the MTP constraint. Finally, we say that $P_{\text {Greedy }}(\mathrm{Q})$ is the approximation given by this greedy algorithm and recall that the true upper bound is $\bar{P}_{\mathcal{G}}^{\Phi}(\mathrm{Q})$. We observe that $P_{\text {Greedy }}(\mathrm{Q}) \leq \bar{P}_{\mathcal{G}}^{\Phi}(\mathrm{Q})$. Furthermore, Nemhauser et al. 1978 tells us the following:

$$
P_{\text {Greedy }}(\mathrm{Q})-P_{\mathcal{P}}(\mathrm{Q}) \geq\left(1-\frac{1}{e}\right)\left(\bar{P}_{\mathcal{G}}^{\Phi}(\mathrm{Q})-P_{\mathcal{P}}(\mathrm{Q})\right)
$$

Combining these and multiplying through gives us the following upper and lower bound on the desired probability.

$$
P_{\text {Greedy }}(\mathrm{Q}) \leq \bar{P}_{\mathcal{G}}^{\Phi}(\mathrm{Q}) \leq \frac{e \cdot P_{\text {Greedy }}(\mathrm{Q})-P_{\mathcal{P}}(\mathrm{Q})}{e-1}
$$

It should be noted that depending on the query and database, it is possible for this upper bound to exceed 1.

\section{Discussion, Future \& Related Work}

We propose the novel problem of constraining open-world probabilistic databases at the schema level, without having any additional ground information over individuals. We introduced a formal mechanism for doing this, by limiting the mean tuple probability allowed in any given completion, and then sought to compute bounds subject to these constraints. We now discuss remaining open problems and related work.

Section 4 showed that there exists a query that is NP-hard to compute exactly, and also presented a tractable algorithm for a class of inversion-free queries. The question remains how hard the other queries are - in particular, is the algorithm presented complete. Is there a complexity dichotomy, that is, a set of syntactic properties that determine the hardness of a query subject to MTP constraints. Questions of this form are a central object of study in probabilistic databases. It has been explored for conjunctive queries [Dalvi and Suciu, 2007], UCQs [Dalvi and Suciu, 2012], and a more general class of queries with negation [Fink and Olteanu, 2016].

The central goal of our work is to find stronger semantics based on OpenPDBs, while still maintaining their desirable tractability. This notion of achieving a powerful semantics while maintaining tractability is a common topic of study. De Raedt and Kimmig 2015 study this problem by using a probabilistic interpretation of logic programs to define a model, leading to powerful semantics but a more limited scope of tractability [Fierens et al., 2015]. Description logics [Nardi et al., 2003] are a knowledge representation formalism that can be used as the basis for a semantics. This is implemented in a probabilistic setting in, for example, probabilistic ontologies [Riguzzi et al., 2012; Riguzzi et al., 2015], probabilistic description logics [Heinsohn, 1994], probabilistic description logic programs [Lukasiewicz, 2005], or the bayesian description logics [Ceylan and Peñaloza, 2014].

Probabilistic databases in particular are of interest due to their simplicity and practicality. Foundational work defines a few types of probabilistic semantics, and provides efficient algorithms as well as when they can be applied [Dalvi and Suciu, 2004; Dalvi and Suciu, 2007; Dalvi and Suciu, 2012]. These algorithms along with practical improvements are implemented as industrial level systems such as MystiQ [Ré and Suciu, 2008], SPROUT [Olteanu et al., 2009], MayBMS [Huang et al., 2009], and Trio which implements the closely related Uncertainty-Lineage Databases [Benjelloun et al., 2007].

Problems outside of simple query evaluation are also points of interest for PDBs, for example the most probable database problem [Gribkoff et al., 2014], or the problem of ranking the top-k results [Ré et al., 2007]. In the context of OpenPDBs in particular, Grohe and Lindner 2018 study the notion of an infinite open world, using techniques from analysis to explore when this is feasible. Borgwardt et al. 2017 study an orthogonal way to introduce constraints on OpenPDBs to make the probability bounds realistic, by adding logical constraints based on an ontology.

Finally, probabilistic databases are closely related to other statistical relational models such as Markov logic networks [Richardson and Domingos, 2006] and probabilistic soft logic [Kimmig et al., 2012]. These models implicitly support the open-world assumption, although inference will not be efficient in general given the large number of random variables induced by the open world.

\section{Acknowledgements}

The authors thank Yitao Liang and YooJung Choi for helpful feedback. This work is partially supported by NSF grants \#IIS1657613, \#IIS-1633857, \#CCF-1837129, DARPA XAI grant \#N66001-17-2-4032, NEC Research and gifts from Intel and Facebook Research.

\section{References}

[Abiteboul et al., 1995] Serge Abiteboul, Richard Hull, and Victor Vianu. Foundations of databases. 1995.

[Benjelloun et al., 2007] Omar Benjelloun, Anish Das Sarma, Alon Y. Halevy, Martin Theobald, and Jennifer Widom. Databases with uncertainty and lineage. The VLDB Journal, 17:243-264, 2007.

[Borgwardt et al., 2017] Stefan Borgwardt, Ismail Ilkan Ceylan, and Thomas Lukasiewicz. Ontology-mediated queries for probabilistic databases. In $A A A I, 2017$.

[Carlson et al., 2010] Andrew Carlson, Justin Betteridge, Bryan Kisiel, Burr Settles, Estevam R. Hruschka, and Tom M. Mitchell. Toward an architecture for never-ending language learning. In $A A A I, 2010$.

[Ceylan and Peñaloza, 2014] İsmail İlkan Ceylan and Rafael Peñaloza. The bayesian description logic $\mathcal{B E} \mathcal{L}$. In Stéphane Demri, Deepak Kapur, and Christoph Weidenbach, editors, Automated Reasoning, pages 480-494, Cham, 2014. Springer International Publishing.

[Ceylan et al., 2016] Ismail Ilkan Ceylan, Adnan Darwiche, and Guy Van den Broeck. Open-world probabilistic databases. In $K R, 2016$.

[Cozman, 2000] Fábio Gagliardi Cozman. Credal networks. Artif. Intell., 120:199-233, 2000. 
[Dalvi and Suciu, 2004] Nilesh N. Dalvi and Dan Suciu. Efficient query evaluation on probabilistic databases. The VLDB Journal, 16:523-544, 2004.

[Dalvi and Suciu, 2007] Nilesh N. Dalvi and Dan Suciu. The dichotomy of conjunctive queries on probabilistic structures. In PODS, 2007.

[Dalvi and Suciu, 2012] Nilesh N. Dalvi and Dan Suciu. The dichotomy of probabilistic inference for unions of conjunctive queries. J. ACM, 59:30:1-30:87, 2012.

[De Raedt and Kimmig, 2015] Luc De Raedt and Angelika Kimmig. Probabilistic (logic) programming concepts. $M a$ chine Learning, 100:5-47, 2015.

[Dong et al., 2014] Xin Luna Dong, Evgeniy Gabrilovich, Geremy Heitz, Wilko Horn, Ni Lao, Kevin Murphy, Thomas Strohmann, Shaohua Sun, and Wei Zhang. Knowledge vault: A web-scale approach to probabilistic knowledge fusion. In The 20th ACM SIGKDD International Conference on Knowledge Discovery and Data Mining, KDD '14, New York, NY, USA - August 24 - 27, 2014, pages 601-610, 2014. Evgeniy Gabrilovich Wilko Horn Ni Lao Kevin Murphy Thomas Strohmann Shaohua Sun Wei Zhang Geremy Heitz.

[Fierens et al., 2015] Daan Fierens, Guy Van den Broeck, Joris Renkens, Dimitar Sht. Shterionov, Bernd Gutmann, Ingo Thon, Gerda Janssens, and Luc De Raedt. Inference and learning in probabilistic logic programs using weighted boolean formulas. TPLP, 15:358-401, 2015.

[Fink and Olteanu, 2016] Robert Fink and Dan Olteanu. Dichotomies for queries with negation in probabilistic databases. ACM Trans. Database Syst., 41:4:1-4:47, 2016.

[Gribkoff et al., 2014] Eric Gribkoff, Guy Van den Broeck, and Dan Suciu. The most probable database problem. Proc. BUDA, 2014.

[Grohe and Lindner, 2018] Martin Grohe and Peter Lindner. Probabilistic databases with an infinite open-world assumption. CoRR, abs/1807.00607, 2018.

[Heinsohn, 1994] Jochen Heinsohn. Probabilistic description logics. In UAI, 1994.

[Hinrichs and Genesereth, 2006] Timothy Hinrichs and Michael Genesereth. Herbrand logic. Technical Report LG-2006-02, Stanford University, 2006.

[Huang et al., 2009] Jiewen Huang, Lyublena Antova, Christoph Koch, and Dan Olteanu. Maybms: a probabilistic database management system. In SIGMOD Conference, 2009.

[Jha and Suciu, 2011] Abhay Jha and Dan Suciu. Knowledge compilation meets database theory: Compiling queries to decision diagrams. Theory of Computing Systems, 52:403-440, 2011.

[Kimmig et al., 2012] Angelika Kimmig, Stephen H. Bach, Matthias Broecheler, Bert Huang, and Lise Getoor. A short introduction to probabilistic soft logic. In NIPS 2012, 2012.
[Lukasiewicz, 2005] Thomas Lukasiewicz. Probabilistic description logic programs. In ECSQARU, 2005.

[Nardi et al., 2003] D. Nardi, Werner Nutt, and Francesco M. Donini. The description logic handbook: Theory, implementation, and applications. In Description Logic Handbook, 2003.

[Nemhauser et al., 1978] G. L. Nemhauser, L. A. Wolsey, and M. L. Fisher. An analysis of approximations for maximizing submodular set functions-i. Mathematical Programming, 14(1):265-294, Dec 1978.

[Olteanu et al., 2009] Dan Olteanu, Jiewen Huang, and Christoph Koch. Sprout: Lazy vs. eager query plans for tuple-independent probabilistic databases. 2009 IEEE 25th International Conference on Data Engineering, pages 640-651, 2009.

[Peters et al., 2014] Shanan E Peters, Ce Zhang, Miron Livny, and Christopher Ré. A machine reading system for assembling synthetic paleontological databases. In PloS one, 2014.

[Ré and Suciu, 2008] Christopher Ré and Dan Suciu. Managing probabilistic data with mystiq: The can-do, the could-do, and the can't-do. In SUM, 2008.

[Ré et al., 2007] Christopher Ré, Nilesh N. Dalvi, and Dan Suciu. Efficient top-k query evaluation on probabilistic data. 2007 IEEE 23rd International Conference on Data Engineering, pages 886-895, 2007.

[Reiter, 1981] Raymond Reiter. On closed world data bases. In Readings in artificial intelligence, pages 119-140. Elsevier, 1981.

[Richardson and Domingos, 2006] Matthew Richardson and Pedro M. Domingos. Markov logic networks. Machine Learning, 62:107-136, 2006.

[Riguzzi et al., 2012] Fabrizio Riguzzi, Elena Bellodi, Evelina Lamma, and Riccardo Zese. Epistemic and statistical probabilistic ontologies. In URSW, 2012.

[Riguzzi et al., 2015] Fabrizio Riguzzi, Elena Bellodi, Evelina Lamma, and Riccardo Zese. Reasoning with probabilistic ontologies. In IJCAI, 2015.

[Roth, 1996] Dan Roth. On the hardness of approximate reasoning. Artificial Intelligence, 82(1-2):273-302, 1996.

[Suchanek et al., 2007] Fabian M. Suchanek, Gjergji Kasneci, and Gerhard Weikum. Yago: A Core of Semantic Knowledge. In 16th International Conference on the World Wide Web, pages 697-706, 2007.

[Suciu et al., 2011] Dan Suciu, Dan Olteanu, R. Christopher, and Christoph Koch. Probabilistic Databases. Morgan \& Claypool Publishers, 1st edition, 2011.

[Van den Broeck and Suciu, 2017] Guy Van den Broeck and Dan Suciu. Query Processing on Probabilistic Data: A Survey. Foundations and Trends in Databases. Now Publishers, August 2017. 